\title{
Screening and Evaluation of Multi Drug Resistance Activity of Various 1,2,4-Triazoles Derivatives through in Vitro Anti tubercular
}

\author{
Jagadeesh Kumar Ega, Ambala Nageswara Rao, Kavitha Siddoju, Boggavarapu Jyothi
}

\begin{abstract}
Isoniazid (Isonicotinic acid hydrazide, INH) has been significantly used to treat Mycobacterium tuberculosis. Introduction of drugs like INH, Rifmapicin, Pyrazinamide and Streptomycin resulted in rapid decline in TB cases worldwide. Several factors lead to the emergence of resistant strains of Mycobacterium tuberculosis. HIV infection also contributed to the escalating burden of tuberculosis. In the present examination 1,2,4-triazole subordinates were planned, incorporated and exposed to in vitro antitubercular screening against Mycobacterium tuberculosis H37Rv.Lipophilicity $(\log P)$ of the compounds were also determined to establish a correlation ship between physicochemical properties and antitubercular activity. Mtb CYP121 and CYP125 are considered to be potential targets for drug design. Binding study of azoles with these enzymes have also been reported. However, enough reports are not available on Mtb CYP-ligand binding requirements to improve the MIC of Azole based antitubercular agents. Hence we conducted the docking study of our synthesized triazoles against both Mtb CYP 121 and CYP125 to establish a correlationship between antitubercular activity and receptor binding interactions. In this paper we discuss about the molecular docking studies of the synthesized mercapto and benzthio 1,2,4-triazole compounds 13-18 with different enzyme target which we have employed.
\end{abstract}

Keywords- 1,2,4-triazoles , Mycobacterium tuberculosis, GOLD, GLIDE CYP121 and CYP125.

\section{INTRODUCTION}

Tuberculosis (TB) will be a overpowering ailment achieved Eventually Tom's perusing the bacillus mycobacterium tuberculosis (Mtb). Following contamination, $\mathrm{m}$. Tuberculosis pathogenesis happens On two periods. Those central phase will be an asymptomatic express that could perseveration to quite a while in the host, known as inactive tbilisi. Thus there is an urgent need for new drugs to treat tuberculosis with special emphasis on shortening the regimen than the current drugs and as well as novel pathway for mechanism of action to treat MDR-TB. ${ }^{1-4}$ Recent studies have shown that nitrogen heterocyclic compounds act as anti tubercular agents by inhibiting P-450 mono-oxygenases. ${ }^{5}$

The genome of Mycobacterium tuberculosis encodes 20 distinctive cytochrome P450 compounds. This

Revised Manuscript Received on July 22, 2019.

Jagadeesh Kumar Ega, Department of Chemistry, Chaitanya Autonomous Post Graduate College, Kishanpura, Hanamkonda, Warangal Urban, Telangana State-506009

Ambala Nageswara Rao, ${ }^{a}$ Department of Chemistry, Chaitanya Autonomous Post Graduate College, Kishanpura, Hanamkonda, Warangal Urban, Telangana State-506009

Kavitha Siddoju, Department of Chemistry, Chaitanya Autonomous Post Graduate College, Kishanpura, Hanamkonda, Warangal Urban, Telangana State-506009

Boggavarapu Jyothi, Department of Chemistry, Swarna Bharathi Institute of Science and Technology, Khammam, Telangana, India recommended the human genome encodes $57 \mathrm{P} 450 \mathrm{~s}$, though Mtb encodes 20 P450s.6-7 Greater CYP in Mtb shows that the life form depends vigorously on P450 catalysts for its survival. Recently reported that the CYP P450s are not involved in the mechanism of resistance. ${ }^{10-21}$

Auto Dock Vina is a generally new open-source program for prescription disclosure, nuclear docking and virtual screening, offering multi-focus capacity with unrivaled and precision.

\section{MATERIALS AND METHOD}

Docking accepts a huge employment in the target prescription structure to the regular furthermore pharmaceutical significances. Evaluation of newly synthesized compounds 13-18 against MDR strain by using Mycobacterium tuberculosis $\left(\mathrm{H}_{37} \mathrm{Rv}\right)$.

Bacterial strain Modified Middlebrook 7H9 Broth, BACTEC MGIT 960 tubes, BACTEC MGIT 960 instrument (BD Biosciences Pvt. Ltd.). Middlebrook 7H9 broth (Difco), Bacto casitone (pancreatic digest of casein; Becton Dickinson), BBL Middlebrook OADC enrichment [(oleic acid, albumin, dextrose, catalase) Becton Dickinson], glycerol (Difco), polysorbate 80 (Difco), sterile water, Alamar blue (Invitogen) and deionised water (MilliQ, Millipore). The suspension prepared was used within 20 minutes for the study.

Antitubercular vulnerability testing was performed utilizing clear level bottomed 96-well microplates. $100 \mu \mathrm{L}$ of media $\mathrm{B}$ was added to each well. At that point $100 \mu \mathrm{L}$ of inoculum was added to each well. The examples were 100, $50,25,12.5,6.25$ and $3.125 \mu \mathrm{g} / \mathrm{mL}$. DMSO was utilized as clear and extra control wells (positive and negative control) were additionally kept to limit the test blunder. Plates were secured with sterile breath seals and kept for hatching at $370 \mathrm{C}$ for seven days. And kept for brooding at $37 \mathrm{oC}$ for $24 \mathrm{~h}$. After hatching for 24 hours, the microplates were imagined to identify the adjustment in shade of the wells. No adjustment in shading (blue) in the wells demonstrated the affectability of Mycobacterium tuberculosis to the test mixes and pink shading showed opposition of living being to them.

Media (A): The media was prepared addition of Glycerol $(0.2 \%)$, Casitone $(0.1 \%)$, Tween $80(0.05 \%)$ and $7 \mathrm{H} 9$ broth $(0.47 \mathrm{~g} / 100 \mathrm{~mL})$ in MilliQ water and made up to the desired volume and sterilized.Media (B): The media was prepared by mixing Glycerol $(0.2 \%)$, Casitone $(0.1 \%)$ and $7 \mathrm{H} 9$ broth $(0.47 \mathrm{~g} / 100 \mathrm{~mL})$ in MilliQ water and made up to desired volume and sterilized. 
Human Liver Microsomes (20 mg protein/mL, BD ultimo Pool tm HLM 150) What's more NADPH recovering framework results a and $\mathrm{b}(1.3 \mathrm{~mm}$ NADP, $3.3 \mathrm{~mm}$ glucose 6-phosphate $0.4 \mathrm{U} / \mathrm{mL}$ glucose 6-phosphate dehydrogenase What's more $3.3 \mathrm{~mm} \mathrm{MgCl} 2$, bd Biosciences) were defrosted to $370 \mathrm{C}$ water shower What's more kept once wet ice in the recent past utilize. Will An $1.5 \mathrm{~mL}$ micro axis tube, $713 \mu \mathrm{L}$ purified water, $50 \mu \mathrm{L}$ NADPH recovering framework result $\mathrm{A}, 10 \mu \mathrm{L}$ NADPH recovering framework result $\mathrm{b}$ What's more $2 \mu \mathrm{L}$ about $5 \mathrm{mM}$ test compound $(10 \mu \mathrm{M}$ last concentration) were included Also blended completely by shutting those tube. The parts in the smaller scale rotator were warmed to $37^{\circ} \mathrm{C}$ for 5 minutes in a hatchery. After brooding, the metabolic response was started by the expansion of $25 \mu \mathrm{L}(0.5 \mathrm{mg})$ Human Liver Microsomes. The blend was reversing the topped cylinder twice and kept in the hatchery at $37^{\circ} \mathrm{C}$. Following 0,30 and an hour, response was ceased by pulling back $200 \mu \mathrm{L}$ from the hatched cylinders and added to miniaturized scale rotator tubes $(1.5 \mathrm{~mL})$ containing $200 \mu \mathrm{L}$ of cold acetonitrile containing $0.2 \mu \mathrm{M}$ propranolol as inner standard. The substance were blended and centrifuged at $12000 \mathrm{rpm}$ for 5 minutes. The supernatant was expelled for investigation and pellet was put away at 200C. The examples at 0,30 and an hour interim were broke down by HPLC utilizing RP-HPLC Microsorb-MV 100 C18 segment $(250 \times 4.6 \mathrm{~mm})$ having molecule estimate $5 \mu \mathrm{m}$. Acetonitrile: oxidan $(0.1 \% \mathrm{HCOOH})$ in slope stream is utilized as portable stage. HPLC top territories were incorporated and communicated and a mean pinnacle zone an incentive for each time point was determined from the copies: Cytotoxicity Screening

The cytotoxicity of the synthesized compounds were assessed by Micro culture Tetrazolium Assay. Reagents are Minimum Essential Medium Eagle (MEM, AT047-10x1L), Fetal Bovine Serum (FBS, RM9970-500mL), Trypsin Phosphate Versene Glucose, Phosphate Buffered Saline, pH 7.1 (PBS, TS1099-10x1L), Antibiotic Antimycotic solution 100x Liquid (A002-20mL), Thiazolyl Blue Tetrazolium Bromide reagent (MTT, AR, RM1131-500mG)and DMSO (AR, Spectrochem) and sterile deionised water (MilliQ, Millipore). Cell cultures are used namely Vero cells (African Green monkey kidney epithelial cells) and HepG2 cells (Human Hepato carcinoma cells)

\section{RESULTS AND DISCUSSIONS}

The Compounds 13a-m were procured by showing pyrazine ring at fifth position and diverse alkyl/aryl substitutions by methods for an amide linkage at fourth position of 1,2,4-triazole ring. Ten mixes showed MIC against Mycobacterium tuberculosis at $50 \mu \mathrm{g} / \mathrm{mL}$. Remaining mixes required $\mathrm{MIC}$ values $\geq 100 \mu \mathrm{g} / \mathrm{mL}$. These mixes similarly shown extraordinary wellbeing profile (CC50 $\geq 300$ $\mu \mathrm{g} / \mathrm{mL}$ ) against Vero in the same way that HepG2 units. When these compounds were docked against mycobacterial CYP 121, the $-\mathrm{SH}$ group of triazole derivatives showed hydrogen bonding interactions with $\mathrm{Thr} 77$ and Ala 167 through a water bridge $\left(\mathrm{H}_{2} \mathrm{O}\right.$ 2584) and resulted in good docking score. In order to enhance the lipophilicity of the triazole derivatives, a 5-methyl group was substituted at the $5^{\text {th }}$ position of the pyrazine ring to afford compounds $14 \mathbf{a}-\mathbf{r}$. Although the LogP of the projected compounds improved but the antitubercular activity was significantly attenuated. Only compound $14 f$ could manage to have MIC at $50 \mu \mathrm{g} / \mathrm{mL}$.

It was also found that the amide bond of compounds drifted away from the plane by an angle of $119-120^{\circ}$ and oriented themselves towards $\mathrm{H}_{2} \mathrm{O}-2761$. This was the major change observed in case of series (14a-r) compounds. Also - $\mathrm{SH}$ group of the triazole ligands displayed H-bond interaction with $\mathrm{H}_{2} \mathrm{O}-2761$. Although this change in geometry facilitated them to interact with $\mathrm{H}_{2} \mathrm{O} 2761$, they lost their interaction with $\mathrm{H}_{2} \mathrm{O} 2584, \mathbf{1 4 f}$ which led to a weaker docking score.

Pyrazine ring was replaced with comparatively hydrophobic 3-phenoxy phenyl moiety at $5^{\text {th }}$ position of the triazole ring, to afford compounds 16a-d. Among these derivatives, 16d has shown antitubercular activity at MIC 25 $\mu \mathrm{g} / \mathrm{mL}$, but unable to show sound safety profile against human hepatocytes $\left(\mathrm{CC}_{50} 93 \mu \mathrm{g} / \mathrm{mL}\right)$ which could be attributed to the presence of hydrophobic diphenyl ether ring. Although compounds 17d and 18c displayed moderate to week safety profile $\left(\mathrm{CC}_{50} 90-300 \mu \mathrm{g} / \mathrm{mL}\right)$ they could manage to have $\mathrm{SI} \geq 10$. Introduction of diphenyl ether ring in these compounds improved their hydrophobic interactions but could not form any H-bonds both in CYP 121 and CYP 125. Compound 18c formed H-bond interaction with Gly 202 in CYP 125 and antitubercular activity.

\section{I. $N$-(3-Mercapto-5-(substituted} pyrazin-4-yl)-4H-1,2,4-triazol-4-yl)amides 12 and 13

13a. $\mathrm{R}=\mathrm{H}, \mathrm{R}^{\mathrm{l}}=\mathrm{CH}_{3}, 13$ b. $\mathrm{R}=\mathrm{H}, \mathrm{R}^{\mathrm{l}}=\mathrm{C}_{2} \mathrm{H}_{5}, 13$ c. $\mathrm{R}=\mathrm{H}$, $\mathrm{R}^{1}=\mathrm{C}_{3} \mathrm{H}_{7}, 13$ d. $\mathrm{R}=\mathrm{H}, \mathrm{R}^{\mathrm{l}}=\mathrm{C}_{4} \mathrm{H}_{9}, 13 \mathrm{e} \cdot \mathrm{R}=\mathrm{H}, \mathrm{R}^{\mathrm{l}}=\mathrm{C}_{5} \mathrm{H}_{11}$, 13f. $\mathrm{R}=\mathrm{H}, \mathrm{R}^{\mathrm{l}}=\mathrm{C}_{6} \mathrm{H}_{13}, 13 \mathrm{~g} . \mathrm{R}=\mathrm{H}, \mathrm{R}^{\mathrm{l}}=2-\mathrm{CH}_{3} \mathrm{Ph}, 13 \mathrm{~h}$. $\mathrm{R}=$ $\mathrm{H}, \mathrm{R}^{\mathrm{l}}=3-\mathrm{OCH}_{3} \mathrm{Ph}, 13 \mathrm{i} . \mathrm{R}=\mathrm{H}, \mathrm{R}^{\mathrm{l}}=4-\mathrm{OCH}_{3} \mathrm{Ph}, 13 \mathrm{j} \cdot \mathrm{R}=\mathrm{H}$, $\mathrm{R}^{\mathrm{l}}=4-\mathrm{F} \mathrm{Ph}, 13 \mathrm{k} . \mathrm{R}=\mathrm{H}, \mathrm{R}^{\mathrm{l}}=4-\mathrm{C}_{2} \mathrm{H}_{5} \mathrm{Ph}, 13 \mathrm{l} . \mathrm{R}=\mathrm{H}, \mathrm{R}^{\mathrm{l}}=4$ $\mathrm{OC}_{2} \mathrm{H}_{5} \mathrm{Ph}, 13 \mathrm{~m} . \mathrm{R}=\mathrm{H}, \mathrm{R}^{\mathrm{l}}=3,4-\mathrm{OCH}_{3} \mathrm{Ph}$

\section{II. $N$-(3-Mercapto-5-aryl-4H-1,2,4-triazol-4-yl) (substituted pyrazin-4-yl)-2-caroxamides 14 and 15}


14a. $\mathrm{R}=\mathrm{CH}_{3}, \mathrm{R}^{1}=\mathrm{CH}_{3}$, 14b. $\mathrm{R}=\mathrm{CH}_{3}, \mathrm{R}^{1}=\mathrm{C}_{2} \mathrm{H}_{5}$, 14c. $\mathrm{R}=\mathrm{CH}_{3}, \mathrm{R}^{1}=\mathrm{C}_{3} \mathrm{H}_{7}$, 14d. $\mathrm{R}=\mathrm{CH}_{3}, \mathrm{R}^{1}=\mathrm{C}_{4} \mathrm{H}_{9}$, 14e. $\mathrm{R}=\mathrm{CH}_{3}$, $\mathrm{R}^{1}=\mathrm{C}_{5} \mathrm{H}_{11}$, 14f. $\mathrm{R}=\mathrm{CH}_{3}, \mathrm{R}^{1}=\mathrm{C}_{6} \mathrm{H}_{13}$, 14g. $\mathrm{R}=\mathrm{CH}_{3}, \mathrm{R}^{1}=$ $\mathrm{Ph}$, 14h. $\mathrm{R}=\mathrm{CH}_{3}, \mathrm{R}^{1}=2-\mathrm{CH}_{3} \mathrm{Ph}$, 14i. $\mathrm{R}=\mathrm{CH}_{3}, \mathrm{R}^{1}=$ 4- $\mathrm{CH}_{3} \mathrm{Ph}, 14 \mathbf{j} . \mathrm{R}=\mathrm{CH}_{3}, \mathrm{R}=3-\mathrm{OCH}_{3} \mathrm{Ph}, 14 \mathrm{k} . \mathrm{R}=\mathrm{CH}_{3}$, $\mathrm{R}^{1}=4-\mathrm{OCH}_{3} \mathrm{Ph}, 14 \mathrm{l} . \mathrm{R}=\mathrm{CH}_{3}, \mathrm{R}^{1}=2-\mathrm{Cl} \mathrm{Ph}, 14 \mathrm{~m} . \mathrm{R}=\mathrm{CH}_{3}$, $\mathrm{R}^{1}=4-\mathrm{Cl} \mathrm{Ph,} \mathrm{14n.} \mathrm{R}=\mathrm{CH}_{3}, \mathrm{R}^{1}=4-\mathrm{F} \mathrm{Ph}, \quad 14 o \cdot \mathrm{R}=\mathrm{CH}_{3}$, $\mathrm{R}^{1}=-\mathrm{CH}_{2} \mathrm{O} \mathrm{Ph}, 14$ p. $\mathrm{R}=\mathrm{CH}_{3}, \mathrm{R}^{1}=4-\mathrm{C}_{2} \mathrm{H}_{5} \mathrm{Ph}, 14 \mathrm{q} . \mathrm{R}=$ $\mathrm{CH}_{3}, \mathrm{R}^{1}=4-\mathrm{OC}_{2} \mathrm{H}_{5} \mathrm{Ph}, 14 \mathrm{r} . \mathrm{R}=\mathrm{CH}_{3}, \mathrm{R}^{1}=3,4-\mathrm{OCH}_{3} \mathrm{Ph}$. 15a. $\mathrm{R}=\mathrm{H}$, 15b. $\mathrm{R}=2-\mathrm{CH}_{3}, 15 \mathrm{c} . \mathrm{R}=4-\mathrm{CH}_{3}, 15 d$. $\mathrm{R}=$ $3-\mathrm{OCH}_{3}$, 15e. $\mathrm{R}=4-\mathrm{OCH}_{3}, 15 f . \mathrm{R}=2-\mathrm{Cl}, 15 \mathrm{~g}$. $\mathrm{R}=$ 4-Cl.

III.

3-(Benzylthio)-4-(2,4-dichlorophenyl)-5-(3-phenoxyp henyl)-4H-1,2,4-triazole $16 \mathrm{~d}$

IV.

3-Methoxy- $N$-(3-(5-methylpyrazine-2-yl)-5-oxo-1H-1, 2,4-traizol- 4- $(5 \mathrm{H})$ yl)benzamide $17 \mathrm{c}$

V.

4-(\{[3-Substituted-1H-pyrazol-4-yl]methylidene $\}$ amin o)-5- [(substituted)methyl]-1,2,4- triazole-3-thiols (Schiff bases) 18

\begin{tabular}{|c|c|c|c|c|}
\hline \multirow{2}{*}{$\begin{array}{l}\text { Comp } \\
\text { d }\end{array}$} & \multicolumn{2}{|c|}{ Docking Score $^{\text {a }}$} & \multicolumn{2}{|c|}{ Residue Interaction ${ }^{\mathrm{b}}$} \\
\hline & $\begin{array}{l}\text { CYP } \\
125\end{array}$ & CYP 121 & CYP 125 & CYP 121 \\
\hline 13a & -5.73 & -5.61 & SH-597 $\mathrm{H}_{2} \mathrm{O}$ & SH-2584 $\mathrm{H}_{2} \mathrm{O}$ \\
\hline 13b & -5.3 & -5.01 & Nil & Amide-NH-2584 $\mathrm{H}_{2} \mathrm{O}$ \\
\hline $13 \mathrm{c}$ & -5.31 & -5.22 & SH-597 $\mathrm{H}_{2} \mathrm{O}$ & Tz-NH-2584 $\mathrm{H}_{2} \mathrm{O}$ \\
\hline 13d & -5.17 & -5.03 & Nil & Nil \\
\hline $13 \mathrm{e}$ & -5.14 & -5.5 & Nil & Tz-NH-2584 $\mathrm{H}_{2} \mathrm{O}$ \\
\hline $13 f$ & -5.14 & -5.5 & Nil & Nil \\
\hline $13 \mathrm{~g}$ & -5.74 & -7.53 & Nil & Nil \\
\hline 13h & -5.82 & -5.31 & Nil & Nil \\
\hline 13i & -5.82 & -7.56 & $\mathrm{SH}-597 \mathrm{H}_{2} \mathrm{O}$ & Nil \\
\hline $13 \mathbf{j}$ & -5.97 & -7.61 & Nil & Nil \\
\hline $13 \mathbf{k}$ & -5.79 & -7.79 & SH-597 $\mathrm{H}_{2} \mathrm{O}$ & Nil \\
\hline 131 & -5.89 & -7.66 & Nil & Nil \\
\hline $13 m$ & -6.31 & -4.97 & $\mathrm{SH}-597 \mathrm{H}_{2} \mathrm{O}$ & Nil \\
\hline $14 a$ & -5.73 & -6.24 & Nil & SH-2761 $\mathrm{H}_{2} \mathrm{O}$ \\
\hline $14 b$ & -3.83 & -6.18 & Nil & SH-2761 H2O \\
\hline $14 \mathrm{c}$ & -5.43 & -5.2 & Nil & SH-2761 H2O \\
\hline 14d & -5.46 & -5.15 & Nil & SH-2761 H2O \\
\hline $14 \mathrm{e}$ & -5.41 & -3.62 & $\begin{array}{l}\text { Amide-597H2O and } \\
\text { Pyz-Gly202 }\end{array}$ & SH-2761 H2O \\
\hline $14 \mathrm{f}$ & -5.65 & -3.23 & SH-597 H2O & Tz-NH-2584 H2O \\
\hline $14 \mathrm{~g}$ & -5.14 & -6.06 & Nil & SH-2761 H2O \\
\hline $14 \mathrm{~h}$ & -6.05 & -6.56 & Nil & SH-2761 H2O \\
\hline $14 \mathbf{i}$ & -6.20 & -5.96 & Nil & SH-2761 H2O \\
\hline $14 \mathrm{j}$ & -6.08 & -5.67 & Methoxy-Gly202 & SH-2761 H2O \\
\hline $14 k$ & -5.7 & -5.85 & Amide-597H2O & SH-2761 H2O \\
\hline 141 & -6.37 & -7.02 & SH-597 H2O & SH-2761 H2O \\
\hline $14 m$ & -5.71 & -6.56 & Nil & SH-2761 H2O \\
\hline $14 n$ & -5.49 & -5.69 & SH-597 H2O & SH-2761 H2O \\
\hline 140 & -6.3 & -6.76 & SH-597 H2O & SH-2761 H2O \\
\hline $14 p$ & -6.16 & -5.95 & $\begin{array}{l}\text { SH-597 H2O and } \\
\text { Pyz-Gly202 }\end{array}$ & SH-2761 H2O \\
\hline $14 q$ & -6.04 & -6.07 & SH-597 H2O & Tz-NH-2761 H2O \\
\hline $14 r$ & -6.18 & -4.41 & Methoxy-Gly202 & Amide- $\mathrm{C}=\mathrm{O}-2761 \mathrm{H} 2 \mathrm{O}$ \\
\hline $15 \mathbf{a}$ & -4.34 & -4.85 & Nil & $\begin{array}{l}\text { Pyz- NH-2761 H2O, SH- } \\
\text { Gly-385 }\end{array}$ \\
\hline $15 \mathrm{~g}$ & -6.05 & -6.58 & SH-597 H2O & SH-2761 H2O \\
\hline $16 \mathbf{a}$ & -5.97 & -4.7 & $\begin{array}{c}\text { Phenyllle97,Met264 } \\
\text {; } \\
\text { SH-Gly202 }\end{array}$ & Nil \\
\hline $16 \mathrm{~b}$ & -6.77 & -6.34 & Nil & Nil \\
\hline $16 \mathrm{c}$ & -6.95 & -5.94 & S-Val11 & Nil \\
\hline 16d & -7.9 & -6.71 & S-Val11 & Nil \\
\hline 17d & -8.46 & -3.64 & -S-Gly202,Ile97 & Nil \\
\hline $18 \mathrm{c}$ & -5.92 & -6.38 & $\mathrm{Tz}-\mathrm{C}=\mathrm{O}-597 \mathrm{H} 2 \mathrm{O}$ & $\begin{array}{l}\text { Tz-C }=\mathrm{O}-\mathrm{H} 2 \mathrm{O} \\
2584, \text { Amide- } \\
\mathrm{C}=\mathrm{O}-\text { Gly-385 }\end{array}$ \\
\hline
\end{tabular}

Table-I: Molecular docking data of synthesized selective compounds in CYP 125 and CYP 121 enzyme

1. ${ }^{\mathrm{a}}$ Molecular docking studies with binding score in Schrodinger, ${ }^{b}$ Interactions of molecules with the amino acids in receptor CYP125 and CYP121 through hydrogen bonding. $\mathrm{T} z=$ triazole. 
Screening and Evaluation of Multi Drug Resistance Activity of Various 1,2,4-Triazoles Derivatives through in Vitro Anti tubercular

Table-II: Biological activities of synthesized compounds

\begin{tabular}{|c|c|c|c|}
\hline Compd & $\begin{array}{c}\text { Anti Tubercular } \\
\text { activity MIC }^{\text {a }} \\
(\mu \mathrm{g} / \mathrm{mL})\end{array}$ & $\begin{array}{l}\text { Vero }(\mathrm{CC50}) \\
(\mu \mathrm{g} / \mathrm{mL})\end{array}$ & $\begin{array}{l}\text { HepG2(CC50 } \\
)^{\mathrm{c}} \\
(\mu \mathrm{g} / \mathrm{mL})\end{array}$ \\
\hline 13a & 50 & $>300$ & $>300$ \\
\hline 13b & 50 & $>300$ & $>300$ \\
\hline $13 c$ & 50 & $>300$ & $>300$ \\
\hline 13d & $>100$ & $>300$ & $>300$ \\
\hline $13 \mathrm{e}$ & $>100$ & $>300$ & $>300$ \\
\hline $13 f$ & 50 & $>300$ & $>300$ \\
\hline $13 \mathrm{~g}$ & 100 & $>300$ & $>300$ \\
\hline 13h & $>100$ & $>300$ & $>300$ \\
\hline $13 \mathbf{i}$ & 50 & $>300$ & $>300$ \\
\hline 13j & $>100$ & $>300$ & $>300$ \\
\hline 13k & 50 & $>300$ & $>300$ \\
\hline 131 & 100 & $>300$ & $>300$ \\
\hline $13 \mathrm{~m}$ & $>100$ & $>300$ & $>300$ \\
\hline $14 a$ & $>100$ & $>300$ & $>300$ \\
\hline $14 \mathrm{~b}$ & $>100$ & $>300$ & $>300$ \\
\hline $14 c$ & $>100$ & $>300$ & $>300$ \\
\hline 14d & $>100$ & $>300$ & $>300$ \\
\hline $14 \mathrm{e}$ & $>100$ & $>300$ & $>300$ \\
\hline $14 f$ & 50 & $>300$ & $>300$ \\
\hline $14 \mathrm{~g}$ & 100 & $>300$ & $>300$ \\
\hline $14 \mathrm{~h}$ & $>100$ & $>300$ & $>300$ \\
\hline $14 \mathbf{i}$ & 100 & $>300$ & $>300$ \\
\hline $14 \mathrm{j}$ & $>100$ & $>300$ & $>300$ \\
\hline $14 k$ & 100 & $>300$ & $>300$ \\
\hline 141 & 100 & $>300$ & $>300$ \\
\hline $14 \mathrm{~m}$ & 50 & $>300$ & $>300$ \\
\hline $14 n$ & 50 & $>300$ & $>300$ \\
\hline 140 & 100 & $>300$ & $>300$ \\
\hline $14 p$ & 100 & $>300$ & $>300$ \\
\hline $14 q$ & 50 & $>300$ & $>300$ \\
\hline $14 r$ & 100 & $>300$ & $>300$ \\
\hline $15 a$ & $>100$ & $>300$ & $>300$ \\
\hline $15 \mathrm{~g}$ & 20 & $>300$ & $>300$ \\
\hline $16 \mathbf{a}$ & 25 & $>300$ & $>300$ \\
\hline $16 \mathrm{~b}$ & 50 & $>300$ & $>300$ \\
\hline $16 \mathrm{c}$ & 50 & $>300$ & $>300$ \\
\hline 16d & $>100$ & $>300$ & $>300$ \\
\hline 17d & 50 & $<50$ & $<50$ \\
\hline $18 \mathrm{c}$ & 25 & $>300$ & $>300$ \\
\hline
\end{tabular}

${ }^{\mathrm{a}}$ Antitubercular activity against $\mathrm{H}_{37} \mathrm{Rv}$ strain, ${ }^{\mathrm{b}}$ Cytotoxicity evaluation by MTT assay method using Vero (African green monkey epithelial cells), ${ }^{\mathrm{c}}$ cytotoxicity evaluation by MTT assay method using HepG2 (hepatic carcinoma cells).

Table- III: Log P of compounds with anti TB MIC $\leq 25$ $\mu \mathrm{g} / \mathrm{mL}$

\begin{tabular}{|l|l|}
\hline Compd & $\log \mathbf{P}^{1}$ \\
\hline $\mathbf{1 5 b}$ & 0.228 \\
\hline $\mathbf{1 5 g}$ & 2.010 \\
\hline $\mathbf{1 6 d}$ & 3.692 \\
\hline $\mathbf{1 7 d}$ & 2.140 \\
\hline $\mathbf{1 8 c}$ & 0.920 \\
\hline
\end{tabular}

${ }^{1}$ Calculated from the capacity factors of compounds at various $\%$ of organic phase used.

Fig-1: Typical plot of antitubercular activity Vs Log P of compounds with $\mathrm{MIC} \leq 25 \mu \mathrm{g} / \mathrm{mL} \mu \mathrm{g} / \mathrm{mL}$ and $\mathrm{SI} \geq 10$

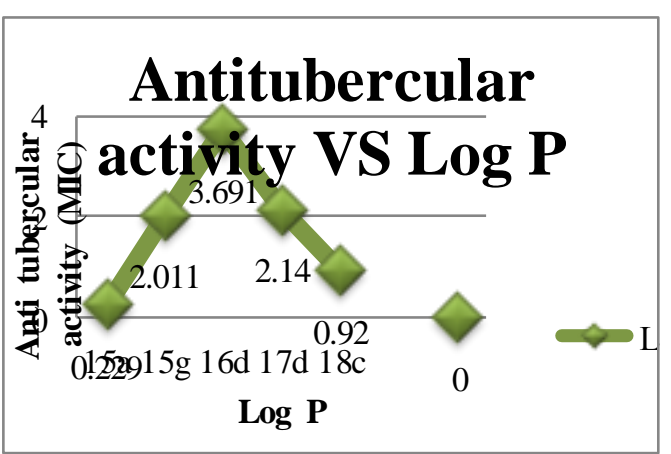

Table-IV: Microsomal stability of compounds with anti TB $\mathrm{MIC} \leq 25 \mu \mathrm{g} / \mathrm{mL}$

\begin{tabular}{|l|l|}
\hline Compd & \% Remaining \\
\hline 15b & 64.46 \\
\hline 15g & 36.36 \\
\hline 16d & 50.78 \\
\hline 17d & 75.49 \\
\hline 18c & 88.39 \\
\hline Testosterone(Ts) & 64.04 \\
\hline
\end{tabular}

${ }^{1}$ Amount of drug remaining (\%) after metabolisation by CYP enzymes after $1 \mathrm{~h}$.

Fig-2: Microsomal stability assay of compounds

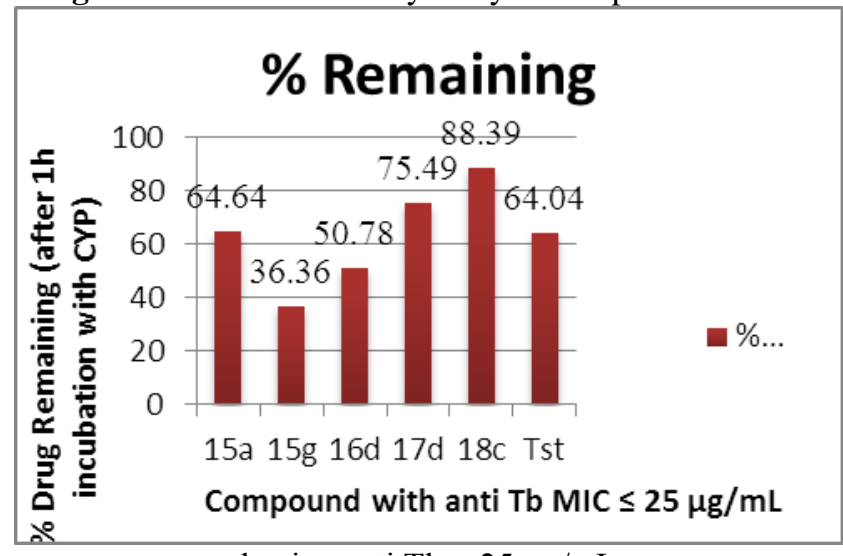

having anti $\mathrm{Tb} \leq 25 \mu \mathrm{g} / \mathrm{mL}$

C: CYP 125 with $\mathbf{1 7 d}$

D: CYP 125 with 18c
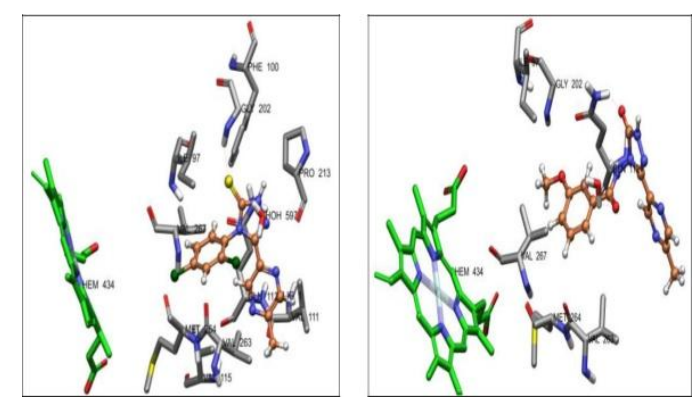

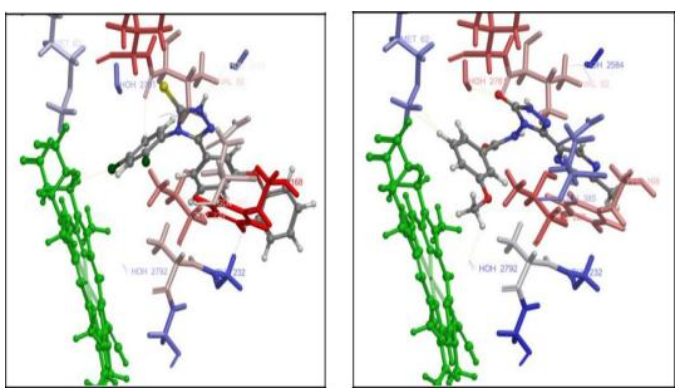

\section{G: CYP 121 with 17d}

\section{H: CYP 121 with 18c}

${ }^{a}$ Antitubercular activity against $\mathrm{H}_{37} \mathrm{Rv}$ strain, ${ }^{\mathrm{b}}$ Cytotoxicity evaluation by MTT assay method using Vero (African green monkey epithelial cells), cytotoxicity evaluation by MTT assay method using HepG2 (hepatic carcinoma cells).

Fig-3: Docked poses of compounds with CYP 125 (PDB:

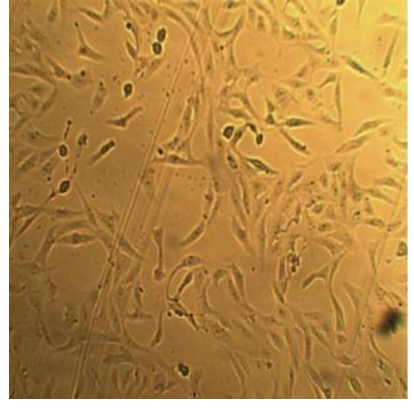

A

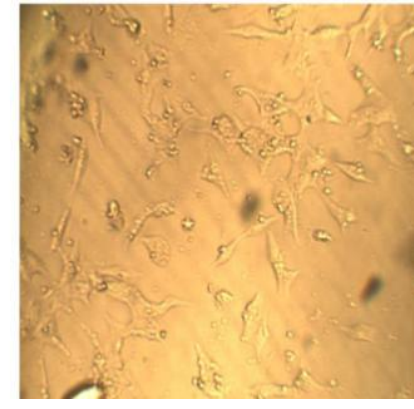

B
Fig-4: Effect of compound 15a at 300 $\mu \mathrm{g} / \mathrm{mL}$ on Vero cells: A. $80 \%$ confluent Vero cells B. After $72 \mathrm{~h}$ of incubation.
C

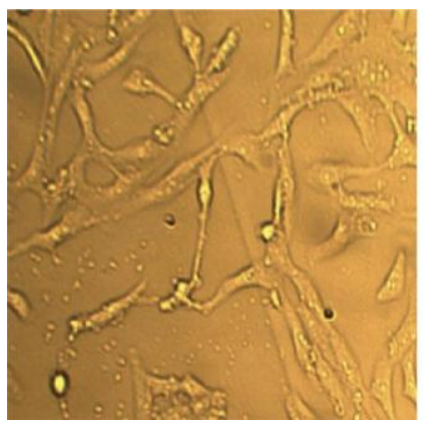

D

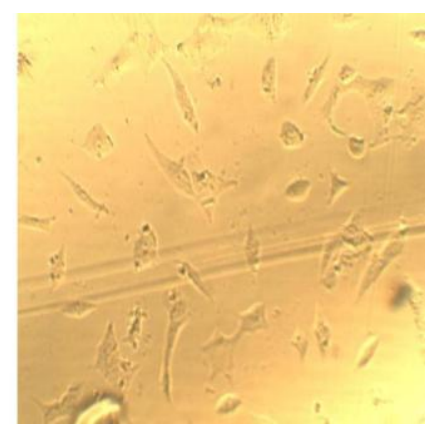

Fig-5: Effect of compound 15a at $300 \mu \mathrm{g} / \mathrm{mL}$ on HepG2 cells: C. $90 \%$ confluent HepG 2 cells. D. After $24 \mathrm{~h}$ Incubation.

\section{CONCLUSION}

With a hope that development of new and effective and concurrent anti tubercular agents, was made to synthesize substituted 1,2,4-traizole derivatives of 13-18 3WI2) and CYP 121 (PDB: 4G2G) receptors of Mtb.

with drug-like characters. We were great for synthesizing number triazole subsidiaries with calculable security profile, worthy metabolic Strength Furthermore hostile to tubercular action under $12.5 \mu \mathrm{g} / \mathrm{ml}$. A standout amongst the exacerbates might have been Indeed going animated against MDR strain for mycobacterium tuberculosis toward $25 \mu \mathrm{g} / \mathrm{ml}$. Our endeavor to depict those relationship the middle of Different parameters in docking score Furthermore $\log \mathrm{P}$ values need not yielded fancied majority of the data of the degree foreseen. We feel that there will be further degree will investigate 1,2,4-triazoles clinched alongside point of interest. This might additionally help should land at An All the more serious SAR.

\section{ACKNOWLEDGEMENTS}

Authors are thankful to the director NCL Pune , CDRI Lucknow for facilitating to progress the part of our research .

\section{REFERENCES}

1. Bouzard D, Dicesare P, Essiz M, Jacquet J P, Ledoussal B, Remuzon P, Kessler R E \& Fung Tome J, J Med Chem, Volume 35, (1992), pp.518.

2. Roma G, Braccio M D, Grossi G, Mattioli F \& Ghia M, Eur J Med Chem, Volume 35, (2000), pp.1021.

3. Gorecki D K J \& Hawes E M, J Med Chem, Volume 20, (1977), pp. 124.

4. Sriram D, Senthilkumar P, Dinakaran M, Yogeeswari P \& Nagaraja V, J Med Chem, Volume 50,(2007), pp.6232.

5. Massari S, Daelemans D, Barreca M L, Knezevich A, Sabatini S, Cecchetti V, Marcello A, Pannecouque C \& Tabarrini O, J Med Chem, Volume 53, (2010), pp.641.

6. Badawneh M, Ferrarini P L, Calderone V, \& Testai L, J Med Chem, Volume 36, (2001), pp.925.

7. Marco-Contelles J, Leon R, Rios R, Guglietta A, \& Villarroya M, J Med Chem, Volume 49,(2006), pp.7610.

8. Tsuzuki Y, Tomita K, Sato Y, Kashimoto S \& Chiba K, Bioorg Med Chem Lett, Volume 14,(2004), pp.3189.

9. Xia Song M, Zheng C J, Deng X Q, Sun L P, Wu Y, Hong L, Li Y J, Liu Y, Wei, Z Y, Jin M J \& Piao H R, Eur J Med Chem, Volume 60, (2013), pp.376.

10. Barbuceanu S F, Almajan G L, Saramet I, Draghici C, Tarcomnicu A I \& Bancescu G, Eur J Med Chem, Volume 44, (2009), pp.4752.

11. Gurupadaswamy H D, Girish V, Kavitha C V \& Khanum S A, Eur J Med Chem, Volume 63, (2013), 536.

12. Sharma P K, Chandak N,Kumar P, Sharma C \& Aneja K R, Eur J Med Chem, Volume 46, (2011), pp.1425.

13. Al-Omary F A M, Hassan G S, El-Messery S M \& El-Subbagh H I Eur J Med Chem, Volume 47, (2012), pp.65.

14. Mahran A M, Ragab S Sh, Hashem A I, Ali M M \& Nada A A, Eur J Med Chem, Volume 90, (2015), 568.

15. Indian Pharmacopoeia, Microbiological assay and test, ed. Vol. II,(1996), A-100.

16. T.J Mosmann. J Immunol Meth. (1983), Volume 65, pp. 55-63.

17. Virender Singh \& Ravi Shankar, Bioorganic Chemistry, Volume 71, (2017), pp.30-54.

18. Rafi Haider, European Journal of Medicinal Chemistry, Volume 125, (2017), 143-189.

19. Mikhail A Prezent \& Igor V Zavarzin, Mendeleev Communications, Volume 27, Number 2, (2017), pp.169-171.

20. Jagadeesh .E, Pradeep Kumar. Ch, International Journal of Modern Trends in Engineering and Research . Volume 4, Number 11, (2017), pp. 40-45.

21. Jagadeesh kumar.E , Kumara Swamy.J , Kavitha .S, International Journal of Modern Trends in Engineering and Research . Volume 04, Number 11, (2017), pp. 215-217.iciently testing technical. 